\title{
ON THE MIXED FRACTIONAL BROWNIAN MOTION
}

\author{
MOUNIR ZILI
}

Received 3 October 2005; Revised 24 March 2006; Accepted 24 March 2006

The mixed fractional Brownian motion is used in mathematical finance, in the modelling of some arbitrage-free and complete markets. In this paper, we present some stochastic properties and characteristics of this process, and we study the $\alpha$-differentiability of its sample paths.

Copyright (c) 2006 Mounir Zili. This is an open access article distributed under the Creative Commons Attribution License, which permits unrestricted use, distribution, and reproduction in any medium, provided the original work is properly cited.

\section{Introduction}

It is well known that the fractional Brownian motion of Hurst parameter $H \in] 0 ; 1[$ is a centered Gaussian process $B^{H}=\left\{B_{t}^{H}, t \geq 0\right\}$, defined on a probability space $(\Omega, F, \mathbb{P})$, with the covariance function

$$
\operatorname{Cov}\left(B_{t}^{H}, B_{s}^{H}\right)=\frac{1}{2}\left(s^{2 H}+t^{2 H}-|t-s|^{2 H}\right) .
$$

If $H=1 / 2, B^{H}$ is the ordinary Brownian motion denoted by $B=\left\{B_{t}, t \geq 0\right\}$. Among the properties of this process, we recall the following:

(i) $B_{0}^{H}=0 \mathbb{P}$-almost surely;

(ii) for all $t \geq 0, \mathbb{E}\left(\left(B_{t}^{H}\right)^{2}\right)=t^{2 H}$;

(iii) the increments of $B^{H}$ are stationary and self-similar with order $H$;

(iv) the trajectories of $B^{H}$ are almost surely continuous and not differentiable (see [7]).

Let us take $a$ and $b$ as two real constants such that $(a, b) \neq(0,0)$.

Definition 1.1. A mixed fractional Brownian motion (MFBM) of parameters $a, b$, and $H$ is a process $M^{H}=\left\{M_{t}^{H}(a, b) ; t \geq 0\right\}=\left\{M_{t}^{H} ; t \geq 0\right\}$, defined on the probability space $(\Omega, F, \mathbb{P})$ by

$$
\forall t \in \mathbb{R}_{+}, \quad M_{t}^{H}=M_{t}^{H}(a, b)=a B_{t}+b B_{t}^{H},
$$


where $\left(B_{t}\right)_{t \in \mathbb{R}_{+}}$is a Brownian motion, and $\left(B_{t}^{H}\right)_{t \in \mathbb{R}_{+}}$is an independent fractional Brownian motion of Hurst parameter $H$.

This process has been introduced by Cheridito [3] to present a stochastic model of the discounted stock price in some arbitrage-free and complete financial markets. This model is the process $\left(X_{t}^{H}(a, b)\right)_{t \in[0 ; 1]}$ defined by

$$
X_{t}^{H}(a, b)=X_{0}^{H}(a, b) \exp \left(v t+\sigma M_{t}^{H}(a, b)\right),
$$

where $\nu, \sigma$ are constants, $a$ is a strictly positive constant, $b=1$, and $M^{H}(a, b)$ is a mixedfractional Brownian motion of parameters $a, b$, and $H$.

Samuelson [11] has presented and treated the particular process $\left(X_{t}^{1 / 2}(1,0)\right)_{t \in[0 ; 1]}$. Yet, this model has several deficiencies; notably it does not exhibit the long-range dependence property.

That is why many authors, for example Cutland et al. [4], have proposed and studied a fractional version of the Samuelson model, able to account for the possibility of longrun nonperiodic statistical dependence in stock price returns. This fractional model is the stochastic process $\left(X_{t}^{H}(0,1)\right)_{t \in[0 ; 1]}$ with $\left.H \in\right] 1 / 2 ; 1[$. But this model has also some deficiencies; for example, in 2001, Cheridito has shown that such model admits arbitrage. And we recall that intuitively, the existence of an arbitrage is a sign of lack of equilibrium in the market: no real market equilibrium can exist in the long run if there are arbitrages there (see $[8])$.

In view of the advantage of the Cheridito model, this paper presents some stochastic properties and characteristics of the MFBM and is organized as follows. In Section 2, we give some stochastic general properties of the mixed fractional Brownian motion. Section 3 deals with the correlation between the increments and Section 4 treats the Hölder continuity of the sample paths of the process. In Section 5 , we study the $\alpha$-differentiability of the trajectories of the MFBM. This type of differentiability, introduced by Kolwankar and Gangal [6] and studied by Ben Adda and Cresson [1], permits to surpass the difficulty of studying the kinematics and geometric structure of nondifferentiable processes.

\section{The main properties}

It is easy to check the following properties.

Lemma 2.1. The MFBM $\left(M_{t}^{H}(a, b)\right)_{t \in \mathbb{R}_{+}}$satisfies the following properties:

(i) $M^{H}$ is a centered Gaussian process;

(ii) for all $t \in \mathbb{R}_{+}, \mathbb{E}\left(\left(M_{t}^{H}(a, b)\right)^{2}\right)=a^{2} t+b^{2} t^{2 H}$;

(iii) one has that

$$
\begin{gathered}
\forall s \in \mathbb{R}_{+}, \forall t \in \mathbb{R}_{+}, \\
\operatorname{Cov}\left(M_{t}^{H}(a, b), M_{s}^{H}(a, b)\right)=a^{2}(t \wedge s)+\frac{1}{2}\left[b^{2}\left(t^{2 H}+s^{2 H}-|t-s|^{2 H}\right)\right],
\end{gathered}
$$

where $t \wedge s=1 / 2(t+s-|t-s|)$;

(iv) the increments of the MFBM are stationary. 
Notation 2.2. Let $\left(X_{t}\right)_{t \in \mathbb{R}_{+}}$and $\left(Y_{t}\right)_{t \in \mathbb{R}_{+}}$be two processes defined on the same probability space $(\Omega, F, \mathbb{P})$. The notation $\left\{X_{t}\right\} \triangleq\left\{Y_{t}\right\}$ will mean that $\left(X_{t}\right)_{t \in \mathbb{R}_{+}}$and $\left(Y_{t}\right)_{t \in \mathbb{R}_{+}}$have the same law.

Lemma 2.3. For any $h>0,\left\{M_{h t}^{H}(a, b)\right\} \triangleq\left\{M_{t}^{H}\left(a h^{1 / 2}, b h^{H}\right)\right\}$.

This property will be called the mixed-self-similarity.

Proof. For fixed $h>0$, the processes $\left\{M_{h t}^{H}(a, b)\right\}$ and $\left\{M_{t}^{H}\left(a h^{1 / 2}, b h^{H}\right)\right\}$ are Gaussian and centered. Therefore, one only has to prove that they have the same covariance function.

But, for any $s$ and $t$ in $\mathbb{R}_{+}$, since $B$ and $B^{H}$ are independent, we have

$$
\begin{aligned}
\operatorname{Cov}\left(M_{h t}^{H}(a, b), M_{h s}^{H}(a, b)\right) & =\mathbb{E}\left(M_{h t}^{H}(a, b) M_{h s}^{H}(a, b)\right) \\
& =a^{2} \mathbb{E}\left(B_{h t} B_{h s}\right)+a b\left(\mathbb{E}\left(B_{h t} B_{h s}^{H}\right)+\mathbb{E}\left(B_{h t}^{H} B_{h s}\right)\right)+b^{2} \mathbb{E}\left(B_{h t}^{H} B_{h s}^{H}\right) \\
& =a^{2} h(t \wedge s)+\frac{1}{2}\left[b^{2} h^{2 H}\left(t^{2 H}+s^{2 H}-|t-s|^{2 H}\right)\right] \\
& =\operatorname{Cov}\left(M_{t}^{H}\left(a h^{1 / 2}, b h^{H}\right), M_{s}^{H}\left(a h^{1 / 2}, b h^{H}\right)\right) .
\end{aligned}
$$

Then the lemma is proved.

Theorem 2.4. For all $H \in] 0 ; 1\left[\backslash\{1 / 2\}, a \in \mathbb{R}\right.$ and $b \in \mathbb{R} \backslash\{0\},\left(M_{t}^{H}(a, b)\right)_{t \in \mathbb{R}_{+}}$is not $a$ Markovian process.

Proof. The process $M^{H}$ is a centered Gaussian and for all $t>0$,

$$
\operatorname{Cov}\left(M_{t}^{H}, M_{t}^{H}\right)=a^{2} t+b^{2} t^{2 H}>0
$$

Then, if $M^{H}$ was a Markovian process, according to Revuz and Yor [9], for all $s<t<u$, we would have

$$
\operatorname{Cov}\left(M_{s}^{H}, M_{u}^{H}\right) \operatorname{Cov}\left(M_{t}^{H}, M_{t}^{H}\right)=\operatorname{Cov}\left(M_{s}^{H}, M_{t}^{H}\right) \operatorname{Cov}\left(M_{t}^{H}, M_{u}^{H}\right) .
$$

In the particular case where $s=1 / 2, t=1$, and $u=3 / 2$, we will have

$$
\operatorname{Cov}\left(M_{1 / 2}^{H}, M_{3 / 2}^{H}\right) \operatorname{Cov}\left(M_{1}^{H}, M_{1}^{H}\right)=\operatorname{Cov}\left(M_{1 / 2}^{H}, M_{1}^{H}\right) \operatorname{Cov}\left(M_{1}^{H}, M_{3 / 2}^{H}\right),
$$

which is equivalent to the following equations:

$$
\begin{aligned}
\frac{1}{2}\left[a^{2}\right. & \left.+b^{2}\left(\frac{1}{2^{2 H}}+\frac{3^{2 H}}{2^{2 H}}-1\right)\right]\left(a^{2}+b^{2}\right) \\
& =\frac{1}{2}\left(a^{2}+b^{2}\right) \times \frac{1}{2}\left[2 a^{2}+b^{2}\left(1+\frac{3^{2 H}}{2^{2 H}}-\frac{1}{2^{2 H}}\right)\right] \\
& \Longleftrightarrow\left(\frac{1}{2^{2 H}}+\frac{3^{2 H}}{2^{2 H}}-1\right)=\frac{1}{2}\left(1+\frac{3^{2 H}}{2^{2 H}}-\frac{1}{2^{2 H}}\right) \\
& \Longleftrightarrow 3+3^{2 H}-3 \times 2^{2 H}=0,
\end{aligned}
$$


4 On the mixed fractional Brownian motion

but it is easy to check that for all $H \in] 0 ; 1[\backslash\{1 / 2\}$,

$$
3+3^{2 H}-3 \times 2^{2 H} \neq 0 .
$$

We deduce that $M^{H}$ is not a Markovian process.

\section{Correlation between the increments}

Notation 3.1. Let $X$ and $Y$ be two random variables defined on the same probability space $(\Omega, F, \mathbb{P})$. We denote the correlation coefficient $\rho(X, Y)$ by

$$
\rho(X, Y)=\frac{\operatorname{Cov}(X, Y)}{\sqrt{V(X)} \sqrt{V(Y)}} .
$$

We can check the following lemma without any difficulty.

Lemma 3.2. One has

$$
\begin{gathered}
\forall s \in \mathbb{R}_{+}, \forall t \in \mathbb{R}_{+}, \forall h \in \mathbb{R}_{+}, 0<h \leq t-s, \\
\rho\left(M_{t+h}^{H}-M_{t}^{H}, M_{s+h}^{H}-M_{s}^{H}\right)=\frac{b^{2}}{2\left(a^{2} h+b^{2} h^{2 H}\right)}\left[(t-s+h)^{2 H}-2(t-s)^{2 H}+(t-s-h)^{2 H}\right] .
\end{gathered}
$$

Corollary 3.3. For all $a \in \mathbb{R}$ and $b \in \mathbb{R} \backslash\{0\}$, the increments of $\left(M_{t}^{H}(a, b)\right)_{t \in \mathbb{R}_{+}}$are positively correlated if $1 / 2<H<1$, uncorrelated if $H=1 / 2$, and negatively correlated if $0<H<1 / 2$.

Proof. If $H>1 / 2$ (resp., $H<1 / 2$ ), from the convexity (concavity) of the function $x \mapsto x^{2 H}$, we derive

$$
\forall x \in \mathbb{R}_{+}, \forall h \in \mathbb{R}_{+} \backslash\{0\}, \quad(x+h)^{2 H}-2 x^{2 H}+(x-h)^{2 H}>0[<0] .
$$

Consequently, using Lemma 3.2, if $H>(1 / 2)$ (resp., $H=1 / 2, H<1 / 2$ ),

$$
\rho\left(M_{t+h}^{H}-M_{t}^{H}, M_{s+h}^{H}-M_{s}^{H}\right)>0[=0,<0] .
$$

Comments on Lemma 3.2 and Corollary 3.3. (i) If $H>1 / 2$ (resp., $H<1 / 2$ ), if $a \neq 0, b_{1}$ and $b_{2}$ are two real constants such that $\left|b_{1}\right| \leq\left|b_{2}\right|\left[\left|b_{1}\right| \geq\left|b_{2}\right|\right]$, then

$$
\begin{gathered}
\forall s \in \mathbb{R}_{+}, \forall t \in \mathbb{R}_{+}, \forall h \in \mathbb{R}_{+}, 0<h \leq t-s, \\
\rho\left(M_{t+h}^{H}\left(a, b_{1}\right)-M_{t}^{H}\left(a, b_{1}\right), M_{s+h}^{H}\left(a, b_{1}\right)-M_{s}^{H}\left(a, b_{1}\right)\right) \\
\leq \rho\left(M_{t+h}^{H}\left(a, b_{2}\right)-M_{t}^{H}\left(a, b_{2}\right), M_{s+h}^{H}\left(a, b_{2}\right)-M_{s}^{H}\left(a, b_{2}\right)\right) .
\end{gathered}
$$

Then, if $H>(1 / 2)$ (resp., $H<(1 / 2))$,

(1) the smaller (larger) $|b|$ is, the less correlated the increments of $M^{H}$ are,

(2) the larger (smaller) $|b|$ is, the more correlated the increments of $M^{H}$ are. 
(ii) If $H>1 / 2$ (resp., $H<(1 / 2)$ ), if $b \neq 0, a_{1}$ and $a_{2}$ are two real constants such that $\left|a_{1}\right| \leq\left|a_{2}\right|\left[\left|a_{1}\right| \geq\left|a_{2}\right|\right]$, then

$$
\begin{gathered}
\forall s \in \mathbb{R}_{+}, \forall t \in \mathbb{R}_{+}, \forall h \in \mathbb{R}_{+}, 0<h \leq t-s, \\
\rho\left(M_{t+h}^{H}\left(a_{2}, b\right)-M_{t}^{H}\left(a_{2}, b\right), M_{s+h}^{H}\left(a_{2}, b\right)-M_{s}^{H}\left(a_{2}, b\right)\right) \\
\leq \rho\left(M_{t+h}^{H}\left(a_{1}, b\right)-M_{t}^{H}\left(a_{1}, b\right), M_{s+h}^{H}\left(a_{1}, b\right)-M_{s}^{H}\left(a_{1}, b\right)\right) .
\end{gathered}
$$

Then, if $H>1 / 2$ (resp., $H<(1 / 2)$ ),

(1) the smaller (larger) $|a|$ is, the more correlated the increments of $M^{H}$ are,

(2) the larger (smaller) $|a|$ is, the less correlated the increments of $M^{H}$ are.

Consequence. In the modelling of a certain phenomenon, we can choose $H, a$, an $b$ suitably in such a manner that $\left\{M_{t}^{H}(a, b)\right\}$ permits to obtain a good model, taking the sign and the level of correlation between the increments of this phenomenon into account.

We now make the following definition.

Definition 3.4. Let $\left\{X_{t}, t \in \mathbb{R}_{+}\right\}$be a process with stationary trajectories and $(r(n))_{n \in \mathbb{N}^{*}}$ the sequence defined by

$$
\forall n \in \mathbb{N}^{\star}, \quad r(n)=\mathbb{E}\left(X_{n+1} X_{1}\right) .
$$

The process $X$ is called long-range dependent if and only if

$$
\sum_{n \in \mathbb{N}^{\star}} r(n)=+\infty
$$

Remark 3.5. Since $\left\{X_{t}, t \in \mathbb{R}_{+}\right\}$is a process with stationary trajectories,

$$
\forall s \in \mathbb{R}_{+}, \forall n \in \mathbb{N}^{\star}, \quad r(n)=\mathbb{E}\left(X_{n+s} X_{s}\right) .
$$

Lemma 3.6. For all $a \in \mathbb{R}$ and $b \in \mathbb{R} \backslash\{0\}$, the increments of $\left(M_{t}^{H}(a, b)\right)_{t \in \mathbb{R}_{+}}$are long-range dependent if and only if $H>1 / 2$.

Proof. For all $n \in \mathbb{N}^{\star}$,

$$
\begin{aligned}
r(n) & =\mathbb{E}\left(\left(M_{n+1}^{H}-M_{n}^{H}\right) M_{1}^{H}\right)=\frac{b^{2}}{2}\left[(n+1)^{2 H}+(n-1)^{2 H}-2 n^{2 H}\right] \\
& =b^{2} H(2 H-1) n^{2 H-2}+n^{2 H-2} \epsilon(n),
\end{aligned}
$$

where $\lim _{n \rightarrow+\infty} \epsilon(n)=0$.

We see that $\sum_{n \in \mathbb{N}^{\star}} r(n)=+\infty$ if and only if $2 H-2>-1$; that is, if and only if $H>1 / 2$. 
6 On the mixed fractional Brownian motion

\section{Hölder-continuity}

Lemma 4.1. For all $T>0$ and $\gamma<1 / 2 \wedge H$, the MFBM has a modification which sample paths have a Hölder-continuity, with order $\gamma$, on the interval $[0 ; T]$.

Proof. According to Kolmogorov's theorem of regularity (see Revuz and Yor [9, page 25]), it suffices to prove that

$$
\forall \alpha>0, \exists C_{\alpha}, \forall(s, t) \in[0 ; T]^{2}, \quad \mathbb{E}\left(\left|M_{t}^{H}-M_{s}^{H}\right|^{\alpha}\right) \leq C_{\alpha}|t-s|^{\alpha(1 / 2 \wedge H)} .
$$

Let $\alpha>0$ and let $s, t \in[0 ; T]$.

Using the stationarity and the mixed-self-similarity (see Lemma 2.3) of the increments of $M^{H}$, we have

$$
\begin{aligned}
\mathbb{E}\left(\left|M_{t}^{H}-M_{s}^{H}\right|^{\alpha}\right) & =\mathbb{E}\left(\left|M_{t-s}^{H}\right|^{\alpha}\right) \\
& =\mathbb{E}\left(\left|M_{1}^{H}\left(a(t-s)^{1 / 2}, b(t-s)^{H}\right)\right|^{\alpha}\right) .
\end{aligned}
$$

First case. If $H \leq 1 / 2$, there are two positive constants $C_{1}$ and $C_{2}$, depending on $\alpha$, such that

$$
\begin{aligned}
\mathbb{E}\left(\left|M_{t}^{H}-M_{s}^{H}\right|^{\alpha}\right) & \leq(t-s)^{\alpha H} \mathbb{E}\left(\left|M_{1}^{H}\left(a(t-s)^{(1 / 2)-H}, b\right)\right|^{\alpha}\right) \\
& \leq(t-s)^{\alpha H}\left[C_{1}|a|^{\alpha}(t-s)^{\alpha((1 / 2)-H)} \mathbb{E}\left(\left|B_{1}\right|^{\alpha}\right)+C_{2}|b|^{\alpha} \mathbb{E}\left(\left|B_{1}^{H}\right|^{\alpha}\right)\right] \\
& \leq C_{\alpha}(t-s)^{\alpha H},
\end{aligned}
$$

where

$$
C_{\alpha}=C_{1}|a|^{\alpha} T^{\alpha((1 / 2)-H)} \mathbb{E}\left(\left|B_{1}\right|^{\alpha}\right)+C_{2}|b|^{\alpha} \mathbb{E}\left(\left|B_{1}^{H}\right|^{\alpha}\right) .
$$

Second case. If $H>(1 / 2)$, there are two positive constants $C_{1}^{\prime}$ and $C_{2}^{\prime}$, depending on $\alpha$, such that

$$
\begin{aligned}
\mathbb{E}\left(\left|M_{t}^{H}-M_{s}^{H}\right|^{\alpha}\right) & \leq(t-s)^{\alpha / 2} \mathbb{E}\left(\left|M_{1}^{H}\left(a, b(t-s)^{H-(1 / 2)}\right)\right|^{\alpha}\right) \\
& \leq(t-s)^{\alpha / 2}\left[C_{1}^{\prime}|a|^{\alpha} \mathbb{E}\left(\left|B_{1}\right|^{\alpha}\right)+C_{2}^{\prime}|b|^{\alpha}(t-s)^{\alpha(H-(1 / 2))} \mathbb{E}\left(\left|B_{1}^{H}\right|^{\alpha}\right)\right] \\
& \leq C_{\alpha}(t-s)^{\alpha / 2},
\end{aligned}
$$

where

$$
C_{\alpha}=C_{1}^{\prime}|a|^{\alpha} \mathbb{E}\left(\left|B_{1}\right|^{\alpha}\right)+C_{2}^{\prime} T^{\alpha(H-(1 / 2))}|b|^{\alpha} \mathbb{E}\left(\left|B_{1}^{H}\right|^{\alpha}\right) .
$$




\section{On the $\alpha$-differentiability of the MFBM}

The following notions have been introduced by Kolwankar and Gangal [6], and studied by Ben Adda and Cresson [1].

Definition 5.1. Let $f$ be a continuous function on $[a ; b]$, and let $\alpha \in] 0 ; 1[$. Call a right (resp., left) local fractional $\alpha$-derivative of $f$ at $t_{0} \in[a ; b]$ the following quantity:

$$
d_{\sigma}^{\alpha} f\left(t_{0}\right)=\Gamma(1+\alpha) \lim _{t \rightarrow t_{0}^{\sigma}} \frac{\sigma\left(f(t)-f\left(t_{0}\right)\right)}{\left|t-t_{0}\right|^{\alpha}}
$$

for $\sigma=+$ (resp., $\sigma=-$ ), where $\Gamma$ is the Euler function.

Definition 5.2. Let $f$ be a continuous function on $[a ; b]$, and let $\alpha \in] 0 ; 1[$. The function $f$ is $\alpha$-differentiable at $t_{0} \in[a ; b]$ if and only if $d_{-}^{\alpha} f\left(t_{0}\right)$ and $d_{+}^{\alpha} f\left(t_{0}\right)$ exist and are equal. In this case, denote by $d^{\alpha} f\left(t_{0}\right)$ the $\alpha$-derivative of $f$ at $t_{0}$.

Remark 5.3. From the previous definition, we obtain the notion of $\alpha$-velocity introduced by Cherbit [2].

Remark 5.4. There are many differences between the so-called fractional derivative of Riemann and Liouville, see Samko et al. [10], and our fractional derivative, introduced in Definition 5.2; we cite just three of them.

(i) First, there is no geometric idea supporting the first-kind derivative notions, from which we understand the difficulties of using them in order to obtain information about the structure of nondifferentiable objects. On the other hand, there is a clear geometrical meaning of our derivative; it gives the local Hölderian behavior of the function, and the critical order of derivation is equal to the Hölder exponent (see [1]).

(ii) Second, the first-kind derivatives are nonlocal on the contrary of our derivative (also on the contrary of the classical derivative).

(iii) Third, contrary to Riemann-Liouville, our derivative of a constant function is zero. This allows to generalize some classical results of analysis to the nondifferentiable case (see [1]).

Theorem 5.5. For all $\alpha \in] 0,1 / 2 \wedge H[$, the sample paths of the MFBM are almost surely $\alpha$-differentiable at every $t_{0} \geq 0$, and

$$
\forall t_{0} \geq 0, \quad \mathbb{P}\left\{d^{\alpha} M_{t_{0}}^{H}=0\right\}=1
$$

Proof. We detail the proof for $\sigma=+$. The proof for $\sigma=-$ is the same.

Using the stationarity and the mixed-self-similarity of the increments of the MFBM, we have for $t>t_{0} \geq 0$,

$$
\begin{aligned}
\frac{M_{t}^{H}-M_{t_{0}}^{H}}{\left(t-t_{0}\right)^{\alpha}} & \triangleq \frac{M_{t-t_{0}}^{H}}{\left(t-t_{0}\right)^{\alpha}} \triangleq\left(t-t_{0}\right)^{-\alpha} M_{1}^{H}\left(a\left(t-t_{0}\right)^{1 / 2}, b\left(t-t_{0}\right)^{H}\right) \\
& \triangleq a\left(t-t_{0}\right)^{1 / 2-\alpha} B_{1}+b\left(t-t_{0}\right)^{H-\alpha} B_{1}^{H} .
\end{aligned}
$$


8 On the mixed fractional Brownian motion

In consequence, if $0<\alpha<1 / 2 \wedge H$,

$$
\begin{aligned}
\mathbb{P}\left\{d_{+}^{\alpha} M_{t_{0}}^{H}=0\right\} & =\mathbb{P}\left\{\lim _{t \rightarrow t_{0}^{+}} \frac{M_{t}^{H}-M_{t_{0}}^{H}}{\left(t-t_{0}\right)^{\alpha}}=0\right\} \\
& =\mathbb{P}\left\{\lim _{t \rightarrow t_{0}^{+}} a\left(t-t_{0}\right)^{1 / 2-\alpha} B_{1}+b\left(t-t_{0}\right)^{H-\alpha} B_{1}^{H}=0\right\}=1 .
\end{aligned}
$$

Theorem 5.6. For all $\alpha \in] 1 / 2 \wedge H ; 1[$, the sample paths of the MFBM are nowhere $\alpha$ differentiable, almost surely.

Proof. For $d>0$, we define the events

$$
A(t)=\left\{\sup _{0 \leq s \leq t}\left|\frac{M_{s}^{H}(a, b)}{s^{\alpha}}\right|>d\right\} .
$$

For any sequence $t_{n} \searrow 0$, we have

$$
A\left(t_{n+1}\right) \subset A\left(t_{n}\right)
$$

thus,

$$
\mathbb{P}\left\{\lim _{n \rightarrow+\infty} A\left(t_{n}\right)\right\}=\lim _{n \rightarrow+\infty} \mathbb{P}\left\{A\left(t_{n}\right)\right\},
$$

and using the mixed-self-similarity of $M^{H}$,

$$
\begin{aligned}
\mathbb{P}\left\{A\left(t_{n}\right)\right\} & \geq \mathbb{P}\left\{\left|\frac{M_{t_{n}}^{H}(a, b)}{t_{n}^{\alpha}}\right|>d\right\} \\
& =\mathbb{P}\left\{\left|a t_{n}^{1 / 2-\alpha} B_{1}+b t_{n}^{H-\alpha} B_{1}^{H}\right|>d\right\} .
\end{aligned}
$$

(i) If $H<1 / 2$, (in this case $\alpha>H$ )

$$
\begin{gathered}
\mathbb{P}\left\{A\left(t_{n}\right)\right\} \geq \mathbb{P}\left\{\left|a t_{n}^{1 / 2-H} B_{1}+b B_{1}^{H}\right|>t_{n}^{\alpha-H} d\right\} \\
\lim _{n \rightarrow+\infty} \mathbb{P}\left\{\left|a t_{n}^{1 / 2-H} B_{1}+b B_{1}^{H}\right|>t_{n}^{\alpha-H} d\right\}=\mathbb{P}\left\{\left|b B_{1}^{H}\right| \geq 0\right\}=1 .
\end{gathered}
$$

(ii) If $H=1 / 2$, (in this case $\alpha>H$ and $\alpha>1 / 2$ )

$$
\begin{gathered}
\mathbb{P}\left\{A\left(t_{n}\right)\right\} \geq \mathbb{P}\left\{\left|a B_{1}+b B_{1}^{H}\right|>t_{n}^{\alpha-H} d\right\}, \\
\lim _{n \rightarrow+\infty} \mathbb{P}\left\{\left|a B_{1}+b B_{1}^{H}\right|>t_{n}^{\alpha-H} d\right\}=\mathbb{P}\left\{\left|a B_{1}+b B_{1}^{H}\right| \geq 0\right\}=1 .
\end{gathered}
$$

(iii) If $H>1 / 2$, (in this case $\alpha>1 / 2$ )

$$
\begin{gathered}
\mathbb{P}\left\{A\left(t_{n}\right)\right\} \geq \mathbb{P}\left\{\left|a B_{1}+b t_{n}^{H-1 / 2} B_{1}^{H}\right|>t_{n}^{\alpha-1 / 2} d\right\} \\
\lim _{n \rightarrow+\infty} \mathbb{P}\left\{\left|a B_{1}+b t_{n}^{H-1 / 2} B_{1}^{H}\right|>t_{n}^{\alpha-1 / 2} d\right\}=\mathbb{P}\left\{\left|a B_{1}\right| \geq 0\right\}=1 .
\end{gathered}
$$


We conclude that for all $\alpha \in] 1 / 2 \wedge H ; 1\left[\right.$, for all $t_{0} \geq 0$,

$$
\mathbb{P}\left\{\limsup _{t \rightarrow t_{0}^{+}}\left|\frac{M_{t}^{H}-M_{t_{0}}^{H}}{\left(t-t_{0}\right)^{\alpha}}\right|=+\infty\right\}=1,
$$

and the theorem is proved.

Open problem. What about the $\alpha$-differentiability of the MFBM in the case where $\alpha=H$ and $H \neq 1 / 2$ ?

\section{Acknowledgments}

The author thanks the anonymous referee, not only for correcting some mistakes, but also for detailed and useful comments, and also Dr. Charles El-Nouty for pointing out the papers $[3,5]$ to his attention. This work was supported by the Tunisian Ministry of Scientific Research, under the research unity Analyse Stochastique, Complexe et Multifractale at the Preparatory Institute for Military Academies at Sousse.

\section{References}

[1] F. Ben Adda and J. Cresson, About non-differentiable functions, Journal of Mathematical Analysis and Applications 263 (2001), no. 2, 721-737.

[2] G. Cherbit, Dimension locale, quantité de mouvement et trajectoire, Fractals, dimension non entière et applications, Masson, Paris, 1967, pp. 340-352.

[3] P. Cheridito, Mixed fractional Brownian motion, Bernoulli 7 (2001), no. 6, 913-934.

[4] N. J. Cutland, P. E. Kopp, and W. Willinger, Stock price returns and the Joseph effect: a fractional version of the Black-Scholes model, Seminar on Stochastic Analysis, Random Fields and Applications (Ascona, 1993), Progress in Probability, vol. 36, Birkhäuser, Basel, 1995, pp. 327-351.

[5] C. El-Nouty, The fractional mixed fractional Brownian motion, Statistics \& Probability Letters 65 (2003), no. 2, 111-120.

[6] K. Kolwankar and A. D. Gangal, Local fractional derivatives and fractal functions of several variables, Proceedings of International Conference on Fractals in Engineering, Archanon, 1997.

[7] B. B. Mandelbrot and J. W. Van Ness, Fractional Brownian motions, fractional noises and applications, SIAM Review 10 (1968), no. 4, 422-437.

[8] B. Øksendal, Stochastic Differential Equations. An Introduction with Applications, 6th ed., Universitext, Springer, Berlin, 2003.

[9] D. Revuz and M. Yor, Continuous Martingales and Brownian Motion, Grundlehren der Mathematischen Wissenschaften [Fundamental Principles of Mathematical Sciences], vol. 293, Springer, Berlin, 1991.

[10] S. G. Samko, A. A. Kilbas, and O. I. Marichev, Fractional Integrals and Derivatives. Theory and Applications, Gordon and Breach Science, Yverdon, 1993.

[11] P. A. Samuelson, Rational theory of warrant pricing, Industrial Management Review 6 (1965), no. $2,13-31$.

Mounir Zili: University Studies Department, Preparatory Institute for Military Academies, Avenue Maréchal Tito, 4029 Sousse, Tunisia

E-mail address: zilimounir@yahoo.fr 


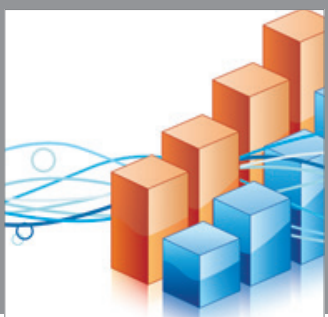

Advances in

Operations Research

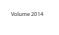

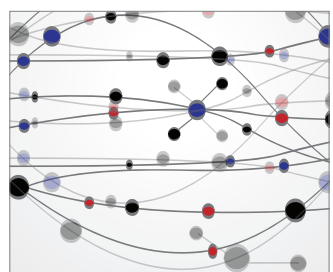

\section{The Scientific} World Journal
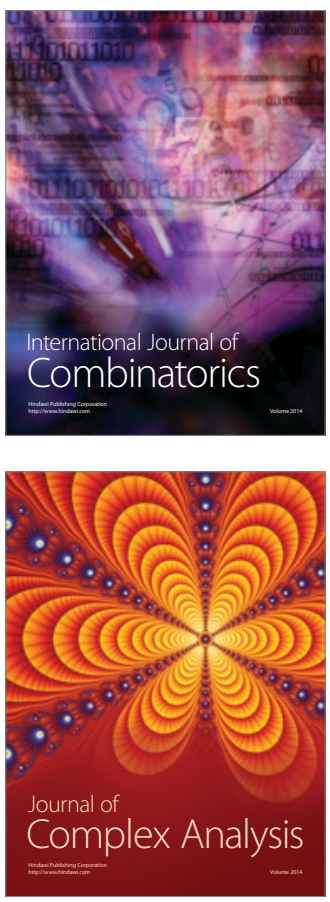

International Journal of

Mathematics and

Mathematical

Sciences
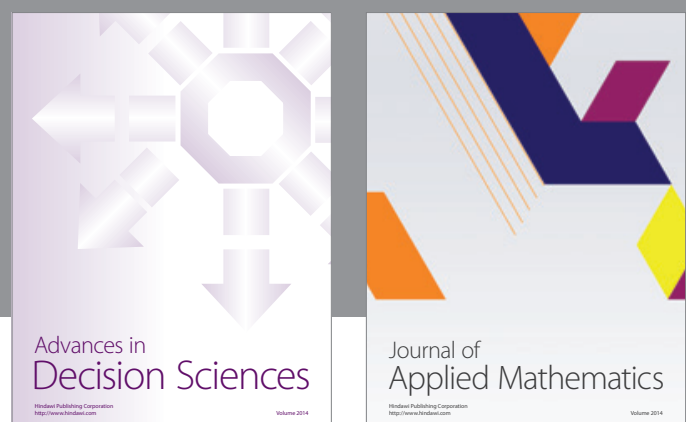

Journal of

Applied Mathematics
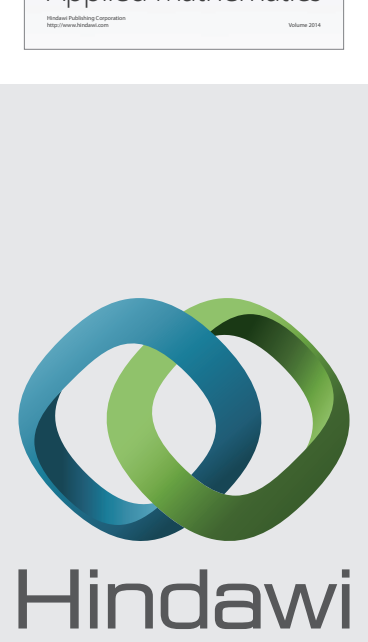

Submit your manuscripts at http://www.hindawi.com
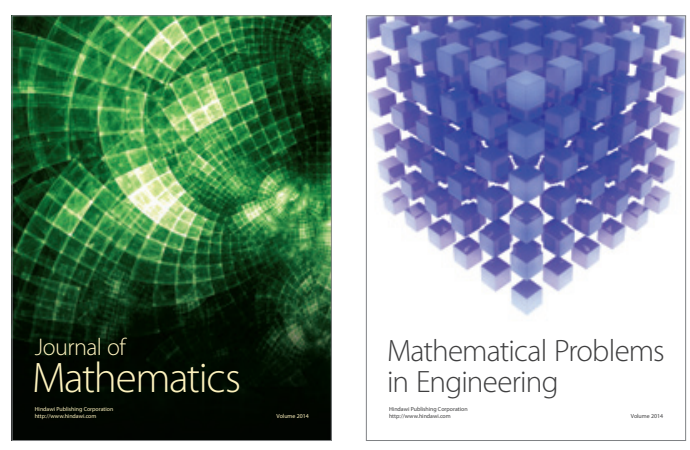

Mathematical Problems in Engineering
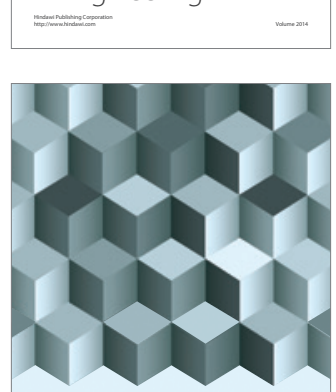

Journal of

Function Spaces
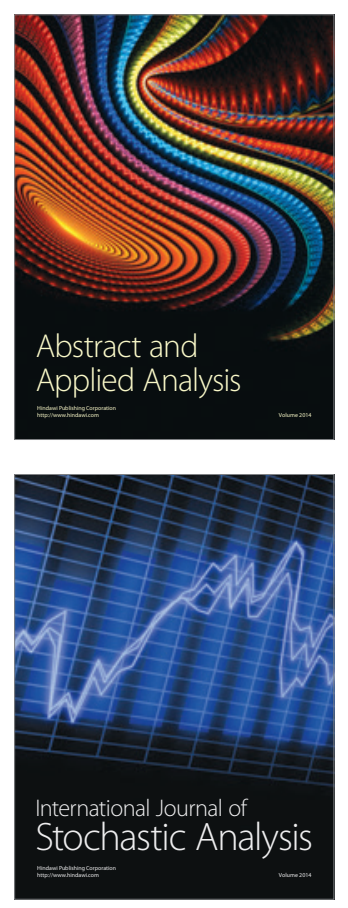

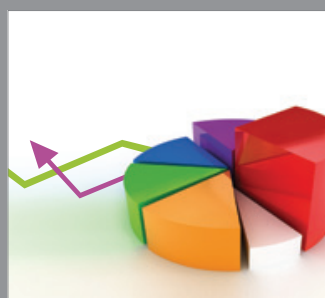

ournal of

Probability and Statistics

Promensencen
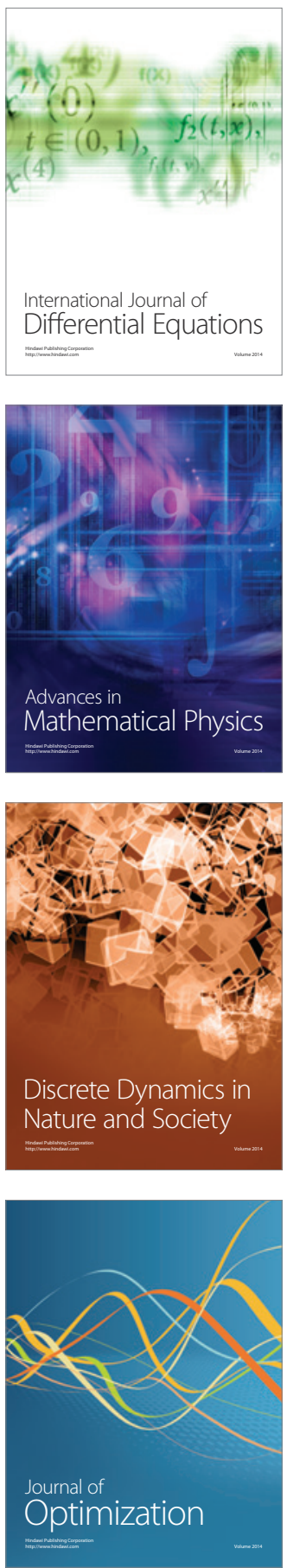